\title{
Pristine cardanol as biobased dopant for polyaniline
}

\author{
Raiane Valenti Gonçalves ${ }^{a}$, Mara Lise Zanini ${ }^{a}$, José Antonio Malmonge ${ }^{b}$, Leila Bonnaud ${ }^{c}$, \\ Nara Regina de Souza Basso ${ }^{\text {a,* }}$ \\ ${ }^{a}$ Faculdade de Química, Pontifícia Universidade Católica do Rio Grande do Sul - PUCRS, Av. Ipiranga, 6681, Porto Alegre, RS, CEP 90619-900 Brazil \\ ${ }^{\mathrm{b}}$ Faculdade de Engenharia, Universidade Estadual Paulista-UNESP, Av. Brasil Sul, 56, Ilha Solteira, SP, CEP 5385-000 Brazil \\ ${ }^{c}$ Laboratory of Polymeric and Composite Materials - Materia Nova Research Center, Avenue Nicolas Copernic, 1, Mons, B-7000 Belgium
}

\section{A R T I C L E I N F O}

\section{Article history:}

Received 24 June 2016

Received in revised form

21 July 2016

Accepted 27 July 2016

Available online 6 August 2016

Keywords:

Cardanol

Polyaniline

Cashew nut liquid shell

Semiconductors

Polymers

\begin{abstract}
A B S T R A C T
Cardanol is a well-known natural phenol bearing a long aliphatic chain. It is obtained from cashew nut shell liquid, a cheap and abundant by-product of the cashew industry. From the viewpoint of conserving the environment and searching for an alternative to petroleum, it represents one of the most promising renewable resources that could be used to replace petro-based phenolic compounds in many applications. Moreover, acidic properties of cardanol broadens the spectrum of its benefits. In this work, the potential of cardanol as a primary dopant to synthesize conductive polyaniline and substitute for conventional acids such as $\mathrm{HCl}$ or DBSA to achieve an environmentally friendly alternative was demonstrated.
\end{abstract}

(c) 2016 Elsevier B.V. All rights reserved.

\section{Introduction}

Polyaniline (PAni) is an intrinsically conducting polymer. Among various conductive polymers, it stands out owing to its chemical stability under ambient conditions and chemical structure [1]. The doping degree of PAni can be adjusted by acid doping, which provides a polymer with suitable conductive property. PAni can be synthesized using an oxidizing agent in acidic medium; several inorganic and organic acids may be used, such as hydrochloric acid $(\mathrm{HCl})$, sulfuric acid $\left(\mathrm{H}_{2} \mathrm{SO}_{4}\right)$, dodecylbenzenesulfonic acid (DBSA) and p-toluenesulfonic acid (PTSA) [2]. These conventional acids are disadvantageous because of the environmental impact caused by the large amount of wastewater containing unreacted acid, inorganic salts, and other by-products produced during the synthesis of PAni [3].

To realize sustainable development and reduce environmental and societal impacts, polymers must be produced from natural sources reagents. Cardanol is a natural phenolic compound with long hydrocarbon side-chain obtained from the purification of the cashew nut liquid shell (CNSL), a by-product of the cashew industry. The functionalization of cardanol's molecules makes it suitable for use as a biomonomer in the synthesis of epoxy and phenolic resins. Moreover, it finds wide applications such as surfactants, plasticizers and antibacterial coatings. The use of

\footnotetext{
* Corresponding author.

E-mail address: nrbass@pucrs.br (N.R. de Souza Basso).
}

cardanol in polymer synthesis is widely exploited because it is considered a sustainable, biodegradable, inexpensive, and natural resource that is widely available [4].

Researchers [5] reported the use of an amphiphilic dopant molecule, 4-[4-hydroxy-2((Z)-pentadec-8-enyl)phenylazo]-benzenesulfonic acid, derived from cardanol for the synthesis of PAni. This dopant could act as structure-directing agent for PAni nanomaterials. Different nanostructures were obtained according to the variation of the [dopant]: [aniline] ratio, and the electrical conductivity values of PAni nanomaterials ranged from $6.0 \times 10^{-6}$ to $6.6 \times 10^{-2} \mathrm{~S} / \mathrm{cm}$. Another research group investigated the action of a natural polymer, tea polyphenol, pre-dissolved in $\mathrm{HCl}$ as a dopant for PAni [6]. The resulting PAni showed conductivity values similar to those of a semiconductive material $\left(8.79 \times 10^{-1} \mathrm{~S} / \mathrm{cm}\right)$, and it showed better solubility and thermal stability than pure PAni. Although these dopants are prepared from renewable raw materials, a large amount of others reagents are used during synthesis, making the process harmful to the environment. Thus, we proposed an environment-friendly approach for the polymerization of PAni using cardanol directly as a dopant. Water was used as the solvent in this synthesis, and the oxidizing agents have low toxicity and their reduction products are lightweight metal salts that can be treated easily.

The present work emphasizes the use of cardanol as a natural primary dopant in the synthesis of PAni and a potential sustainable alternative to the conventional organic acid, DBSA. The selection of this renewable phenol as a dopant was based on the fact that it has an acidic character and long side aliphatic chains; this 
structure is similar to that of DBSA, which also acts as a surfactant. The influence of cardanol on the morphology and electrical property of PAni were investigated and compared to the properties of DBSA-doped PAni, using the same synthesis methodology.

\section{Experimental}

Aniline was purified by distillation before polymerization. All other chemicals were used without further purification. Column chromatography was used to obtain cardanol from technical CNSL. Column chromatography was performed using silica gel 60 (70230 mesh) and a mixture of the solvents chloroform and ethyl acetate (95:5) as an eluent. Doped PAni was synthesized by dispersion polymerization using cardanol as a dopant (PAni-cardanol) and iron (III) chloride $\left(\mathrm{FeCl}_{3}\right)$ and ammonium persulfate (APS) as oxidants, according to the literature [7]. The [dopant]: [aniline] ratio was fixed at 3.3. In a typical polymerization reaction, cardanol $(46.4 \mathrm{mmol})$ and aniline $(14.0 \mathrm{mmol})$ were added to $100 \mathrm{~mL}$ of distilled water. Then, the mixture of oxidants APS $(10.5 \mathrm{mmol})$ and $\mathrm{FeCl}_{3}(6.9 \mathrm{mmol})$ was added to the solution. The reaction occurred at $25{ }^{\circ} \mathrm{C}$ with constant stirring for $5 \mathrm{~h}$. The dark green product was then filtered, washed with distilled water and ethanol to remove oligomers and excess of cardanol, dried for $48 \mathrm{~h}$ at room temperature, and stored in a desiccator. This methodology was also used to produce DBSA-doped PAni (PAni-DBSA). Undoped PAni was prepared by the treatment of $\mathrm{PAni}-\mathrm{HCl}$ with $0.1 \mathrm{M}$ ammonium hydroxide, as described in reference [3]. Technical CNSL was supplied by Vernisul, Brazil.

Field-emission scanning electron microscopy (FESEM) analyses were performed on a microscope Inspect F50. The electrical conductivity was determined by the two probe (undoped PAni) and four-point probe method (Keithley Instruments, model 236, and Multimeter HP34401) on pellet prepared by compacting the PAni powder. The absorption spectra in the infrared region in the $\mathrm{KBr}$ pellets were examined using a Fourier transform infrared spectrometer (FTIR; Perkin Elmer - Spectrum One). UV-visible spectrometry (UV-vis) measurements were performed between 200 and $1100 \mathrm{~nm}$ (HP Hewlett Packard 8453). The samples were dissolved in ethanol. ${ }^{1} \mathrm{H}$-nuclear magnetic resonance $\left({ }^{1} \mathrm{H}\right.$ NMR) measurements were performed on a $300 \mathrm{MHz}$ Inova NMR spectrometer in deuterated chloroform $\left(\mathrm{CDCl}_{3}\right)$.

\section{Results and discussions}

Fig. 1 shows the ${ }^{1} \mathrm{H}$ NMR spectrum of the aromatic and olefin regions of cardanol obtained by purifying technical CNSL. The ${ }^{1} \mathrm{H}$ NMR spectrum confirmed the presence of the aliphatic side chain, because the region between 0.90 and $2.90 \mathrm{ppm}$ indicates the methylene and methyl protons and the signal at 5.00-6.00 ppm is attributable to multiplets of $\mathrm{CH}=\mathrm{CH}$ protons. The region between 6.50 and $7.40 \mathrm{ppm}$ shows the signal of the doublets and triplets that are characteristic of the aromatic groups of cardanol $[8,9]$.

Fig. 2 reveals that polymers prepared with cardanol and DBSA showed characteristic peaks of doped PAni. FTIR (Fig. 2a) showed a peak at $\sim 2915 \mathrm{~cm}^{-1}$ that was attributed to the aliphatic $\mathrm{C}-\mathrm{H}$ stretching of the cardanol or DBSA [8]. The peaks observed at 1566 and $1480 \mathrm{~cm}^{-1}$ were associated with quinoid (Q) and benzenoid (B) stretching rings, respectively. The peaks at $1301 \mathrm{~cm}^{-1}$ and $1113 \mathrm{~cm}^{-1}$ were assigned to the $\mathrm{C}-\mathrm{N}$ deformation of the benzenoid unit and $\mathrm{C}-\mathrm{H}$ bending vibration of the $\mathrm{N}=\mathrm{Q}=\mathrm{N}$ segment respectively [10]. The ratio between the intensity of the peaks $Q$ and $B$ was used to compare the doping efficiency between the samples [11]. Q/B for undoped PAni, PAni-cardanol, and PAni-DBSA was 0.40, 0.86 , and 0.63 , respectively, indicating a higher level of doping in PAni-cardanol. Fig. 2b showed absorption bands at $\sim 310,450$, and $800 \mathrm{~nm}$ that were related to the $\pi-\pi^{*}$ electron transition of the benzenoid segments, polaron- $\pi^{*}$ transition, and $\pi$-polaron transition, respectively [10].

Different experimental parameters such as the type of dopant might influence the morphology of the obtained PAni [2]. In the experimental conditions evaluated in this work, the morphologies achieved exhibited important differences, as shown in Fig. 3: DBSA only produced PAni with an agglomerated morphology, whereas PAni-cardanol showed a mixture of nanofibers and particulates. It was reported in the literature $[5,12]$ that surfactants such as DBSA or cardanol, for instance, can arrange into micelles within water and serve as soft template for the synthesis of PAni materials following a self-assembled process. The presence of nanofibers in PAni-cardanol systems could be explained by the better ability of cardanol to form stable cylindrical micelles when compared to DBSA under the experimental conditions selected and applied in this work. The nanofiber morphology is of interest because it presents several advantages for fabricating nanodevices and for preparing nanoscale electrical connections in highly conducting
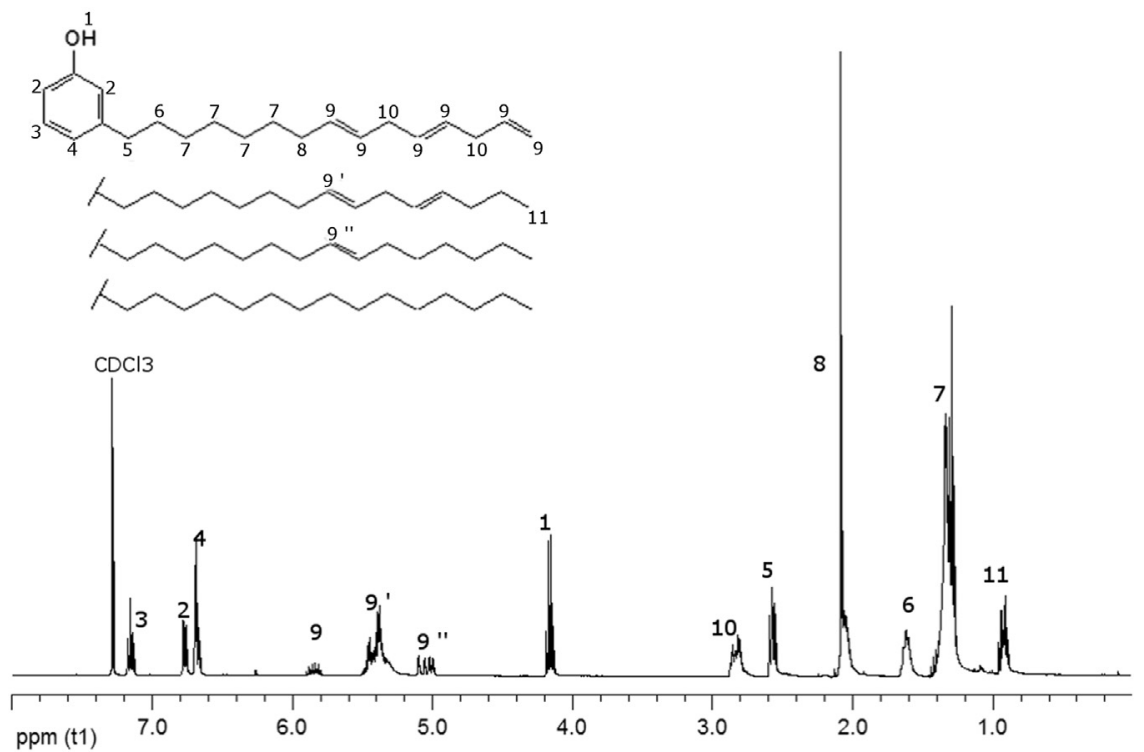

Fig. 1. $1 \mathrm{H}$ NMR spectrum $\left(\mathrm{CDCl}_{3}\right)$ of cardanol obtained from technical CNSL. 

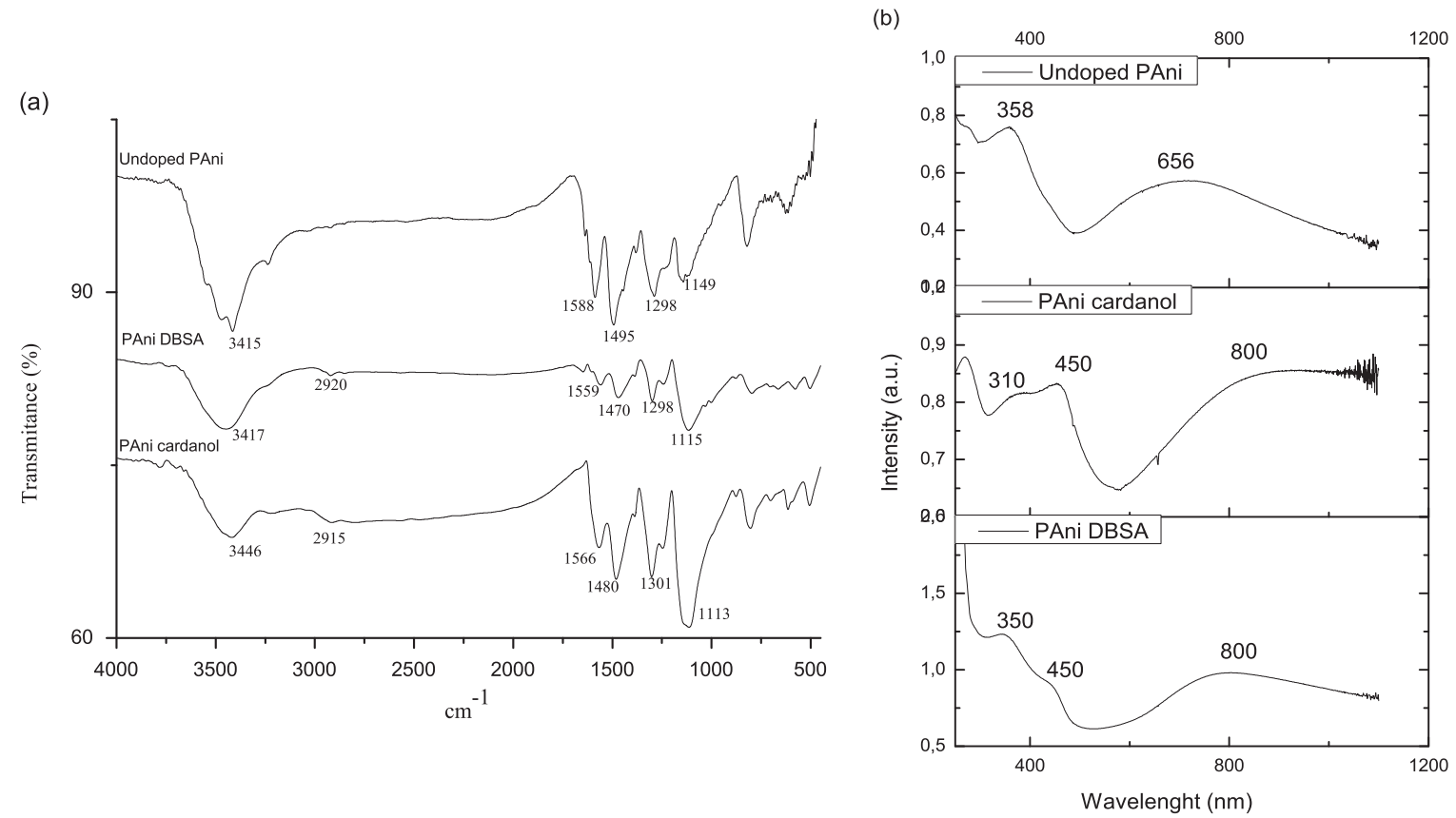

Fig. 2. FTIR spectrum (a) and UV-vis spectra (b) of PAni samples.
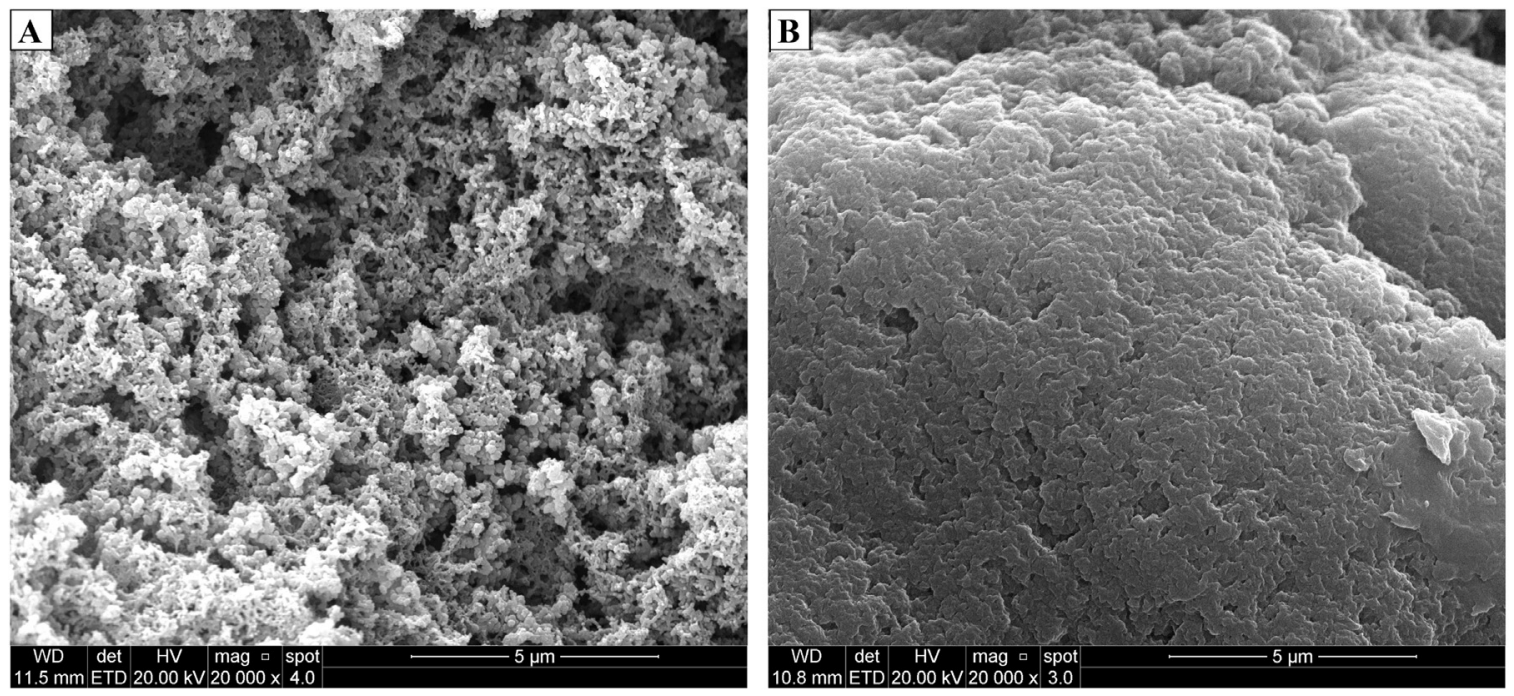

Fig. 3. FESEM images of (a) PAni-cardanol and (b) PAni-DBSA.

Table 1

Electrical conductivity of PAni samples.

\begin{tabular}{ll}
\hline Samples & Conductivity (S/cm) \\
\hline PAni-DBSA & $(6.9 \pm 0.3) \times 10^{-1}$ \\
PAni-cardanol & $(9.7 \pm 0.3) \times 10^{-1}$ \\
Undoped PAni & $(1.2 \pm 0.3) \times 10^{-8}$ \\
\hline
\end{tabular}

polymer composites, owing to its high surface contact area [10].

The action of cardanol as a primary dopant during polymerization was confirmed when the electrical conductivity values of PAni-cardanol were compared with the observed values for undoped PAni, as shown in Table 1. Cardanol as a dopant produced a polymer with the characteristics of a semiconductor material and that showed a conductivity value similar to that of the commonly used organic acid DBSA. The electrical properties might be improved if the experimental parameters are adjusted to produce nanofibers as the major product [5]. More studies under the current investigation are being prepared to produce high-quality PAni-cardanol nanofibers with superior electrical properties.

\section{Conclusion}

In this work, cardanol, an abundant and cheap renewable resource, is found to act efficiently as a primary dopant for PAni, leading to the material exhibiting semiconductor behavior (an electrical conductivity of $9.7 \times 10^{-1} \mathrm{~S} / \mathrm{cm}$ is achieved). Interestingly, the achieved morphology shows the presence of a few nanofibers. The ability of cardanol to allow the formation of nanofibers demonstrates its interesting potential for preparing PAni nanomaterials. These results indicate that cardanol is a promising alternative and could advantageously substitute in a more 
sustainable way the common and less environment-friendly acids $\mathrm{HCl}$ or DBSA to prepare conductive PAni.

\section{Acknowledgements}

This work was partially support by CAPES, Brazil (01845038096) and CNPq, Brazil (grant number-310181/2015-1).

\section{References}

[1] V. Kumar, T. Yokozeki, T. Goto, T. Takahashi, Synthesis and characterization of PANI-DBSA/DVB composite using roll-milled PANI-DBSA complex, Polymer 86 (2016) 129-137.

[2] S. Sinha, S. Bhadra, D. Khastgir, Effect of dopant type on the properties of polyaniline, J. Appl. Polym. Sci. 112 (2009) 3135-3140.

[3] Z. Ma, Q. Shen, J. Kan, Treatment of polyaniline wastewater by Fenton reagent, Asian J. Chem. 25 (14) (2013) 7683-7686.

[4] C. Voirin, S. Caillol, N.V. Sadavarte, B.V. Tawade, B. Boutevin, P.P. Wadgaonkar, Functionalization of cardanol: towards biobased polymers and additives, Polym. Chem. 5 (2014) 3142-3162.
[5] P. Anilkumar, M. Jayakannan, Fluorescent tagged probing agent and structuredirecting amphiphilic molecular design for polyaniline nanomaterials via selfassembly process, J. Phys. Chem. C 111 (2007) 3591-3600.

[6] Q. Yan, M.Y. Wang, Y.H. Wu, Q. Shen, Tea polyphenol as environmentally friendly dopant and thermal stabilizer for polyaniline, Mater. Lett. 170 (2016) 202-204.

[7] J. Jang, J. Bae, K. Lee, Synthesis and characterization of polyaniline nanorods as curing agent and nanofiller for epoxy matrix composite, Polymer 46 (2005) 3677-3684.

[8] R. Srivastava, D. Srivastava, Mechanical, chemical, and curing characteristics of cardanol-furfural-based novolac resin for application in green coatings, J. Coat. Technol. Res. 12 (2) (2015) 303-311.

[9] J. Chen, Z. Liu, X. Li, P. Liu, J. Jiang, X. Nie, Thermal behavior of epoxidized cardanol diethyl phosphate as novel renewable plasticizer for poly(vinyl chloride), Polim. Degrad. Stab. 126 (2016) 58-64.

[10] M.J. Silva, A.O. Sanches, L.F. Malmonge, E.S. Medeiros, M.F. Rosa, C. M. McMahan, J.A. Malmonge, Conductive nanocomposites based on cellulose nanofibrils coated with polyaniline-DBSA via in situ polymerization, Macrom. Symp. 319 (2012) 196-202.

[11] N.R.S. Basso, F. Oliveira, A.P. Graebin, C.S. Moura, F.C. Fim, G.B. Galland, L. Bonnaud, O. Murariu, P. Dubois, Polyaniline nanofibers for in situ MAOcatalyzed polymerization of ethylene, J. Appl. Polym. Sci. 131 (2014) 41197.

[12] H. Yin, J. Yang, Synthesis of high-performance one-dimensional polyaniline nanostructure using dodecylbenzene sulfonic acid as soft template, Mater. Lett. 65 (2011) 850-853. 